\title{
Effectiveness of exercises by telerehabilitation on pain, physical function and quality of life in people with physical disabilities: a systematic review of randomised controlled trials with GRADE recommendations
}

\author{
Jane Fonseca Dias, ${ }^{1}$ Vinicius Cunha Oliveira, ${ }^{2}$ Pollyana Ruggio Tristão Borges, ${ }^{1}$ \\ Fabiana Caetano Martins Silva Dutra, ${ }^{3}$ Marisa Cotta Mancini, ${ }^{1}$ \\ Renata Noce Kirkwood, ${ }^{1}$ Renan Alves Resende $(1),{ }^{4}$ Rosana Ferreira Sampaio ${ }^{5}$
}

\begin{abstract}
- Additional material is published online only. To view, please visit the journal online (http://dx.doi.org/10.1136/ bjsports-2019-101375).

${ }^{1}$ Graduate Program in Rehabilitation Sciences, Universidade Federal de Minas Gerais, Belo Horizonte, Brazil

${ }^{2}$ Pós-Graduação em Reabilitação e Desempenho Funcional, Universidade Federal dos Vales do Jequitinhonha e Mucuri, Diamantina, Minas Gerais, Brazil

${ }^{3}$ Graduate Program in Health Care, Universidade Federal do Triângulo Mineiro, Uberaba, MG, Brazil

${ }^{4}$ Department of Physical Therapy, Graduate Program in Rehabilitation Sciences, Universidade Federal de Minas Gerais, Belo Horizonte, MG, Brazil

${ }^{5}$ Physical Therapy, Universidade Federal de Minas Gerais, Belo Horizonte, Brazil
\end{abstract}

\section{Correspondence to} Dr Rosana Ferreira Sampaio, Physical Therapy, Universidade Federal de Minas Gerais, Belo Horizonte, Brazil; sampaioufmg@gmail.com

Accepted 27 August 2020 Published Online First 15 October 2020

Check for updates

(C) Author(s) (or their employer(s)) 2021. No commercial re-use. See rights and permissions. Published by BMJ.

To cite: Dias JF,

Oliveira VC, Borges PRT,

et al. Br J Sports Med

2021:55:155-162.
ABSTRACT
Objective Investigate whether exercise-based

telerehabilitation improves pain, physical function and quality of life in adults with physical disabilities.

Design Systematic review of randomised controlled trials.

Data sources Searches were performed in AMED, MEDLINE, CINAHL, SPORTDiscus, Embase, PEDro, Cochrane Library and PsycINFO.

Eligibility criteria Trials were considered if they evaluated exercise by telerehabilitation. The population included adults with physical disability. Comparisons were control and other interventions. The outcomes were pain, physical function and quality of life. Study selection, data extraction and analysis followed the protocol registered in PROSPERO (CRD42019122824). GRADE determined the strength of evidence.

Results Forty-eight trials were included in the quantitative analysis. When compared with other interventions, there was high-quality evidence that telerehabilitation was not different to other interventions for pain $(95 \% \mathrm{Cl}$ : -0.4 to 0.1$)$, physical function $(95 \% \mathrm{Cl}$ : -0.2 to 0.2$)$ and quality of life $(95 \% \mathrm{Cl}:-0.1$ to 0.5$)$ at long-term. There was moderate-quality evidence that telerehabilitation was not different to other interventions for physical function $(95 \% \mathrm{Cl}:-0.1$ to 0.5$)$ and quality of life ( $95 \% \mathrm{Cl}:-0.2$ to 0.5$)$ at short-term. However, due to the low-quality evidence and the small number of trials comparing exercise protocols offered by telerehabilitation with control groups, it is still not possible to state the efficacy of telerehabilitation on pain, function and quality of life at short-term and long-term.

Conclusions Exercise by telerehabilitation may be an alternative to treat pain, physical function and quality of life in adults with physical disabilities when compared with other intervention.

\section{INTRODUCTION}

According to the World Report on Disability (WHO 2011), over one billion people live with a disability worldwide, and almost 200 million experience considerable functional limitations. ${ }^{1}$ Healthcare services face challenges to address the needs of people with physical disabilities, ${ }^{2}$ including: patients' physical incapacity to attend treatment centres, absence of caregivers, scarcity of health professionals and limited resources in local communities. Lack of transport to clinical centres can be a particular barrier for people with disability to access care. ${ }^{134}$ Limited access to healthcare services may allow health and quality of life to deteriorate. ${ }^{5}$

To address these challenges, many countries are employing telecommunication technologies as part of the healthcare service. ${ }^{6}$ Telerehabilitation may improve the quality of services by monitoring patients in their own place, mainly in communities far from urban centres. It is also expected to improve cost-effectiveness of interventions. ${ }^{7-9}$ Previous systematic reviews have evaluated the feasibility, efficacy and cost of telerehabilitation for people with different health conditions, and the reviews supported telerehabilitation as an effective alternative to supervised/face-to-face interventions. ${ }^{10-13}$

Exercise is one of the treatments that clinicians can deliver using telerehabilitation. Exercise is costeffective $^{1415}$ and recommended for people with physical disabilities due to musculoskeletal conditions, coronary heart disease, some types of cancer, type 2 diabetes, hypertension, among others. ${ }^{16}$

Conclusions from previous systematic reviews that investigated effectiveness of exercise by telerehabilitation in people with physical disabilities were limited by confounders such as inclusion of poor quality studies (ie, no randomised controlled trials), ${ }^{1718}$ and absence of investigation of effect sizes and the strength of the recommendation. ${ }^{18}$ The aim of this systematic review of randomised controlled trials was to investigate short-term and long-term effectiveness of exercise by telerehabilitation on pain, physical function and quality of life in adults with physical disabilities when compared with control and other interventions. Effect estimates and a rating of the certainty of the current evidence were reported.

\section{METHODS}

Search strategy and inclusion criteria

The present systematic review followed Preferred Reporting Items for Systematic Reviews and MetaAnalyses (PRISMA) ${ }^{19}$ and Cochrane recommendations. ${ }^{20}$ Its protocol was prospectively registered 
at PROSPERO (CRD42019122824). Search strategies were conducted in May 2018 and updated in February 2020 on Allied and Complementary Medicine Database (AMED), Medical Literature Analysis and Retrieval System Online (MEDLINE), Cumulative Index to Nursing and Allied Health Literature (CINAHL), Excerpta Medica dataBASE (Embase), Physiotherapy Evidence Database (PEDro), Cochrane Library, SPORTDiscus and PsycINFO database. There was no date or language restriction. Online supplemental material 1 details the search strategy. The health condition of interest was unlimited to increase sensitivity of our search strategy, avoiding exclusions of potential populations that we were unaware of. In addition, we manually searched identified systematic reviews in the area and specific journals of telemedicine (eg, Journal of Telemedicine and Telecare, and Telemedicine Journal and e-Health) to identify potentially relevant trials.

We included published randomised controlled trials investigating effectiveness of telerehabilitation on pain, physical function and/or quality of life in adults with physical disabilities. Physical disability was defined according to the International Classification of Functioning, Disability and Health (ICF). In the ICF, issues with human functioning are categorised in three interconnected components: impairments are issues in body function or alterations in body structure; activity limitations are issues in executing activities; participation restrictions are issues involving any area of life. Physical disability refers to difficulties encountered by people with health conditions in any or all three components of functioning described above. ${ }^{21}$

Population of interest were adults ( $\geq 18$ years old) with physical disabilities related to any health condition. Telerehabilitation was considered in the current review as any take-home exercise (ie, aerobic exercises and/or kinesiotherapy) provided by telecommunication technologies such as phone calls, video conferences and/or software applications. ${ }^{7}$ We arbitrarily decided to exclude trials investigating virtual reality by telerehabilitation because of the specificity of the theme and costs of the technology. Comparators of interest were control (ie, no intervention, waiting list, placebo or sham) and other interventions (ie, any other active intervention such as traditional rehabilitation at home or in healthcare facilities). Our outcomes of interest were pain, physical function and quality of life. Trials were included if they reported any valid measures of our outcomes of interest such as: Visual Analogue Scale (VAS) or Western Ontario and McMaster Universities Osteoarthritis Index (WOMAC) pain subscale for pain; ${ }^{22} 6$ min walk test (6MWT) or Arthritis SelfEfficacy Scale (AIMS2) subscale for physical function; ${ }^{23}$ and Short Form Health Survey-36 (SF-36 or Minnesota Living with Heart Failure questionnaire for quality of life. ${ }^{24}$ When more than one valid measure was available in the trial for the same outcome, we considered the most consistent measurement instrument across trials included in this review. ${ }^{25-73}$

\section{Study selection}

After searches, retrieved references were exported to the EndNote Reference Manager Software and duplicates were removed. Then, titles and abstracts were screened, and two reviewers independently (JFD and FCMSD) assessed potential full-texts using our eligibility criteria outlined above. Trials fulfilling our eligibility criteria were included in the review. A third reviewer (RFS) solved disagreements.

Two reviewers independently (JFD and PRTB) assessed the quality of included trials using the 0 to 10 PEDro scale (http:// www.pedro.org.au/). The PEDro scale has been shown to have acceptable reliability and validity for rating quality of randomised controlled trials. $^{7475}$ A third reviewer (RFS) solved discrepancies. When available, we used the scores from the PEDro database. ${ }^{76}$

\section{Data extraction}

The two reviewers independently (JFD and PRTB) extracted descriptive and outcome data from included trials, and the third reviewer (RFS) solved discrepancies. Descriptive information included: source of participants; health condition; age; sex; type and dosage for telerehabilitation and comparators; outcomes; and time points. Extracted outcome data included means, standard deviations (SDs) and sample sizes of all groups to investigate short-term and long-term effects. Short-term effect was considered follow-up up to 3 months after baseline, and longterm effect was considered follow-up over 3 months after baseline. When more than one time point was available within the same follow-up period, the one closer to the end of the intervention was considered. If trials investigated more than one type of exercise by telerehabilitation ${ }^{31}$ or more than one comparator, ${ }^{29} 40$ groups were combined as recommended by Cochrane. ${ }^{77}$ Some included trials did not provide SDs and data were imputed from: SEs; ${ }^{26} \mathrm{CIs} ;{ }^{2946}$ P values; ${ }^{4665}$ medians and IQRs; ${ }^{36446164}$ or other trials included in the review that used the same instrument, ${ }^{37}$ following the Cochrane recommendations. ${ }^{77}$ Trials that reported outcome data not normally distributed (ie, mean/SD ratio of less than 2$)^{78}$ and did not provide log-transformed outcome data $2931404856-58687273$ were excluded from the quantitative analyses (ie, meta-analyses), following recommendations. ${ }^{77}$ Online supplemental material 2 details the data extraction.

\section{Study analysis}

Meta-analysis was conducted using random-effects model because of the effects being estimated in the different studies were not identical. The model represents our lack of knowledge about why real or apparent intervention effects differ by considering the differences as if they were random. ${ }^{77}$ For the outcomes of interest, standardised mean differences (SMDs) and 95\% CIs were presented, at first, for overall effect analyses on pain, physical function and quality of life in the forest-plots. The overall effects of telerehabilitation in people with physical disabilities (all health conditions combined) investigated the efficacy of telerehabilitation on outcomes of various functional levels. We chose to do this overall analyses as people with different health conditions may experience similar difficulties across functional levels. ${ }^{79}$ After the overall analyses, subgroup analyses investigated potential impact of specific clinical categories. Trials were categorised following the International Classification of Diseases and Related Health Problems (ICD-10) and grouped into 10 clinical categories (oncology, neurology, cardiovascular, pulmonary, urology, musculoskeletal, postoperative orthopaedic conditions, rheumatological, endocrine and multiple conditions). ${ }^{80}$ Subgroup and sensitivity analyses assessed potential sources of heterogeneity: clinical categories; and methodological quality of included trials (ie, a PEDro score $<6$ out of 10 was considered poor quality), using meta-regression when possible (ie, when at least 10 trials were pooled, following the Cochrane recommendations). ${ }^{77}$ Otherwise, qualitative subgroup analyses were conducted by different clinical categories and removing poor quality trials (ie, when less than 10 trials were pooled). Publication bias was investigated using the funnel plot and the Egger's test when at least 10 trials were pooled. ${ }^{81}$ All analyses were conducted using Comprehensive Meta-analysis software, V.2.2.04 (Biostat, Englewood, New Jersey). Estimated effect 


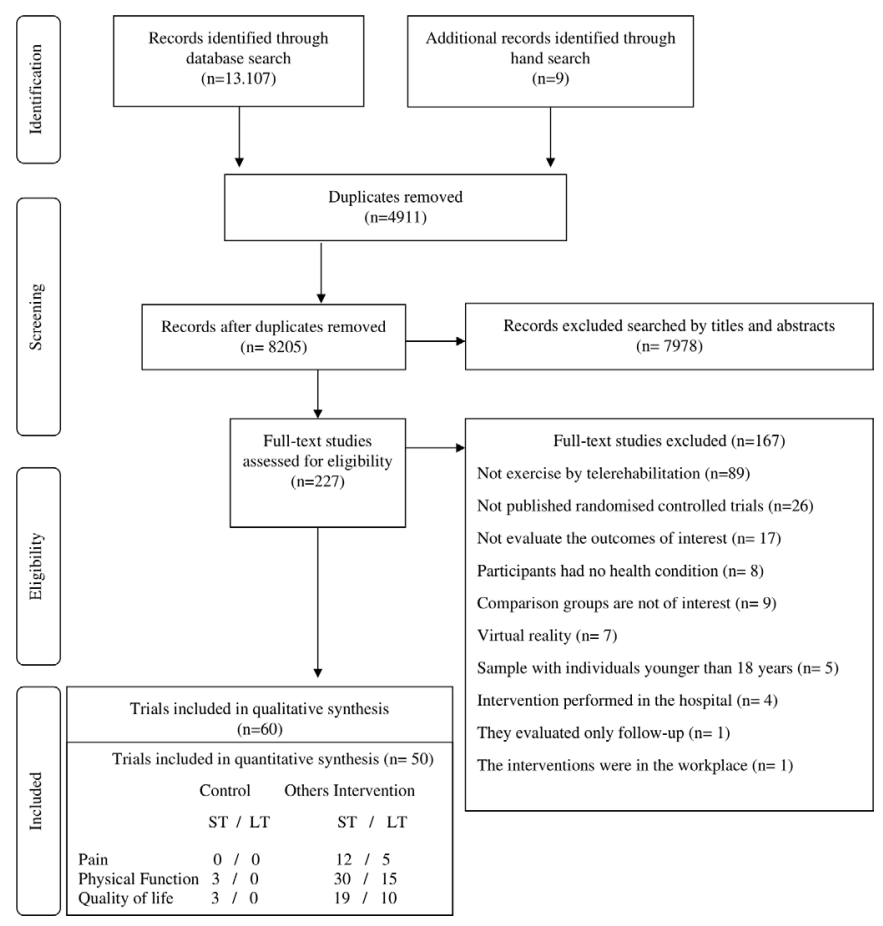

Figure 1 Flow of studies through the review ( $n=60$ original trials included in qualitative synthesis and $n=50$ original trials included in quantitative synthesis). LT, long-term; ST, short-term.

sizes were assessed using Cohen's benchmarks: $d \geq 0.2$ for small; $\mathrm{d} \geq 0.5$ for medium; and $\geq 0.8$ for large effects. ${ }^{82}$

The two reviewers independently (JFD and PRTB) assessed the strength of the recommendation using the GRADE system. ${ }^{83}$ According to the four-level GRADE system, recommendation may range from high to very-low quality. Low levels indicate uncertainty of the estimated effects. In the current review, highquality evidence was downgraded in one point for each of the following issues: imprecision when analysed sample $<400 ;{ }^{84}$ risk of bias when $>25 \%$ of the participants were from trials with a high risk of bias (ie, PEDro score $<6$ out of 10$) ;{ }^{85}$ inconsistency when $\mathrm{I}^{2}$ statistics $>50 \%$ or when pooling was not possible; ${ }^{86}$ and publication bias when pooling $\geq 10$ trials. ${ }^{81}$ A third reviewer (RFS) solved discrepancies between reviewers.

\section{RESULTS}

\section{Study selection}

We identified 8205 references and 60 original trials were included in the review. The main reasons for exclusion of potential full-texts were: no population of interest $(n=13)$; no intervention of interest $(n=100)$; no comparator of interest $(n=9)$; no outcome of interest $(n=17)$; and not published randomised controlled trials $(n=26)$. The flowchart describing trials selection is in figure 1 .

\section{Study characteristics}

Characteristics of included trials and outcome data are presented in online supplemental material 3. All 60 included trials were published between 2002 and 2019. They were conducted in Europe $(n=20,33.3 \%)$, North America $(n=17,28.3 \%)$, Oceania $(n=10,16.6 \%)$, Asia $(n=10,16.6 \%)$, Africa $(n=2,3.3 \%)$ and South America $(n=1,1.6 \%)$. Thirteen trials were conducted in USA and 10 in Australia. In $76 \%$ of the trials $(n=46)$, a single technological resource was used as telerehabilitation (eg, video or telephone). The others combined more than one technology (eg, video and telephone, $\mathrm{n}=4,6.7 \%$; video, telephone and audio, $n=2,3.3 \%$; Internet-based and telephone, $n=2,3.3 \%$ ).

All telerehabilitation exercise programmes included in this review were home-based. The duration ranged from 10 days to 12 months, with weekly frequency and duration of each session ranging from 2 to 7 times and from 20 to $90 \mathrm{~min}$, respectively. Programmes included strength and stretching exercises combined or not with aerobic exercise. Initial evaluation of participants was conducted in all trials. After the initial evaluation, six trials 354553577187 had initial face-to-face contact with participants to establish goals, performed the supervised exercise programme and verified the correct use of telerehabilitation devices. Eight trials 2728344344516588 adopted face-to-face meetings with the telerehabilitation group during the intervention period to conduct sessions supervised by therapists and verified the absence of complications.

Seven trials with 898 participants compared telerehabilitation with control (ie, no intervention, waiting list, placebo or sham), ${ }^{25} 313252-5472$ and 53 trials including 4920 participants compared telerehabilitation with other interventions (ie, traditional rehabilitation at home or in healthcare settings, gym-base exercises, written programmes, usual caremedications and oxygen prescription, medical and other professionals follow-up and encouragement to improve physical activity). ${ }^{26-30} 33-51$ 55-71 73 89-93 Forty one trials reported shortterm effects (ie, $\leq 3$ months after baseline) and 19 reported long-term effects (ie, $>3$ months after baseline). Pain, physical function and quality of life were investigated in 23, 55 and 37 trials, respectively.

\section{Quality of the methods in the included trials}

The quality of the methods in the included trials ranged from 4 to 8 points on the 0 to 10 PEDro scale (table 1 ). All trials reported random allocation, differences between groups, point measures and measures of variability. Forty (66.6\%) out of the 60 included trials scored above 6 points on the PEDro scale. The main reasons for downgrading the methodological quality were lack of therapist blinding $(n=60,100 \%)$, lack of participant blinding $(n=60,100 \%)$, lack of concealed allocation $(n=30$, $50 \%)$ and absence of intention-to-treat analysis $(n=29,48 \%)$.

\section{Effects of telerehabilitation on pain, physical function and quality of life}

We presented our quantitative findings by outcome of interest (data from 50 trials). First, we report the overall effect analyses of telerehabilitation in people with physical disabilities (all health conditions combined) (figure 2). We then categorise effects by subgroups of health conditions categorised according to the ICD-10 were estimated (figure 3 ). In the overall effect analyses, evidence was downgraded due to risk of bias (PEDro score $<6$ ) and /or inconsistency $\left(\mathrm{I}^{2}>50 \%\right)$. We found no evidence of publication bias (ie, Funnel plots and Egger's tests when pooling at least 10 trials are provided in online supplemental material 4).

Overall effects (all health conditions were combined) of telerehabilitation on pain, physical function and quality of life Pain

In the overall effect analyses for pain at long-term, there was high-quality evidence that telerehabilitation was not different to other interventions (SMD: $-0.2 ; 95 \% \mathrm{CI}$ : -0.4 to $0.1 p=0.079$; five trials ${ }^{27} 28304647 ; n=830$ participants). At short-term, the strength of the recommendation was low and very low when 
Review

Table 1 Methodological quality of the included trials using the 0 to 10 PEDro scale. ( $n=60$ original trials). *Trials included in the quantitative analysis $(n=50)$

\begin{tabular}{|c|c|c|c|c|c|c|c|c|c|c|c|}
\hline Study & 2 & 3 & 4 & 5 & 6 & 7 & 8 & 9 & 10 & 11 & $\begin{array}{l}\text { Total } \\
\text { ( } 0 \text { to } 10)\end{array}$ \\
\hline Allen KD, et al $(2010)^{\star 46}$ & Y & Y & Y & $\mathrm{N}$ & $\mathrm{N}$ & N & Y & Y & Y & Y & 7 \\
\hline Ariza-Garcia A, et al (2019)*96 & Y & Y & Y & N & $\mathrm{N}$ & Y & $\mathrm{N}$ & Y & $Y$ & Y & 7 \\
\hline Azma K, et al $(2018)^{* 42}$ & Y & N & Y & N & N & $\mathrm{N}$ & $\mathrm{N}$ & Y & Y & Y & 5 \\
\hline Bennell KL, et al (2017)* & Y & Y & Y & $\mathrm{N}$ & $\mathrm{N}$ & Y & Y & Y & Y & $\mathrm{Y}$ & 8 \\
\hline Bourne $S$, et al $(2017)^{* 55}$ & Y & Y & Y & N & N & Y & Y & Y & Y & Y & 8 \\
\hline Brooks D, et al (2002)*65 & Y & $\mathrm{N}$ & Y & $\mathrm{N}$ & $\mathrm{N}$ & Y & $\mathrm{N}$ & $\mathrm{N}$ & Y & Y & 5 \\
\hline Buhrman M, et al (2004)*25 & Y & N & Y & N & N & N & Y & $\mathrm{N}$ & Y & Y & 5 \\
\hline Calner T, et al $(2017)^{* 47}$ & Y & Y & N & $\mathrm{N}$ & N & $\mathrm{N}$ & $\mathrm{N}$ & $\mathrm{N}$ & $Y$ & Y & 4 \\
\hline Carrion Perez F, et al $(2015)^{* 61}$ & Y & N & Y & N & N & N & N & $\mathrm{N}$ & Y & Y & 4 \\
\hline Chumbler N, et al (2012) $)^{\star 38}$ & Y & Y & Y & N & $\mathrm{N}$ & $\mathrm{N}$ & Y & Y & $Y$ & Y & 7 \\
\hline Conroy SS, et al $(2018)^{* 91}$ & Y & N & Y & N & N & Y & N & N & Y & Y & 5 \\
\hline Coronado RA, et al (2019)*97 & Y & Y & Y & $\mathrm{N}$ & $\mathrm{N}$ & Y & Y & Y & Y & Y & 8 \\
\hline Cuperus $\mathrm{N}$, et al $(2015)^{48}$ & Y & N & Y & N & $\mathrm{N}$ & Y & Y & Y & Y & Y & 7 \\
\hline Damush TM, et al (2003) $)^{\star 28}$ & Y & Y & Y & $\mathrm{N}$ & N & Y & $\mathrm{N}$ & Y & $\mathrm{Y}$ & Y & 7 \\
\hline Demeyer $\mathrm{H}$, et al $(2017)^{56}$ & Y & Y & Y & N & N & $\mathrm{N}$ & Y & Y & Y & Y & 7 \\
\hline Duruturk N and MA Ozkoslu (2019)*87 & Y & Y & Y & $\mathrm{N}$ & $\mathrm{N}$ & $\mathrm{N}$ & Y & $\mathrm{N}$ & Y & Y & 6 \\
\hline Ellis TD, et al (2019)*66 & Y & Y & Y & N & N & Y & Y & Y & Y & Y & 8 \\
\hline Fang J, et al (2019)*88 & Y & $\mathrm{N}$ & Y & $\mathrm{N}$ & $\mathrm{N}$ & $\mathrm{N}$ & $\mathrm{N}$ & $\mathrm{N}$ & Y & Y & 4 \\
\hline Fjeldstad-Pardo C, et al (2018) $)^{40}$ & Y & $\mathrm{N}$ & Y & N & N & Y & Y & N & Y & Y & 6 \\
\hline Frederix I, et al (2015)*11 & Y & $\mathrm{N}$ & Y & $\mathrm{N}$ & $\mathrm{N}$ & Y & Y & Y & Y & Y & 7 \\
\hline Galiano-Castillo $\mathrm{N}$, et al (2017) $)^{* 90}$ & Y & Y & Y & N & $\mathrm{N}$ & Y & Y & Y & Y & Y & 8 \\
\hline Hwang R, et al (2017)*62 & Y & Y & Y & N & N & Y & Y & Y & Y & Y & 8 \\
\hline Iles $R$, et al $(2011)^{* 43}$ & Y & Y & Y & $\mathrm{N}$ & $\mathrm{N}$ & $\mathrm{N}$ & Y & Y & Y & Y & 7 \\
\hline Jackson JC, et al (2012) $)^{* 44}$ & Y & Y & Y & N & N & Y & Y & N & Y & Y & 7 \\
\hline Jansons P, et al $(2017)^{* 67}$ & Y & Y & Y & $\mathrm{N}$ & $\mathrm{N}$ & Y & Y & Y & $Y$ & $Y$ & 8 \\
\hline Kalron $\mathrm{A}$, et al $(2018)^{* 89}$ & Y & Y & Y & N & N & Y & $\mathrm{N}$ & N & Y & Y & 6 \\
\hline Kraal JJ, et al (2014)*35 & Y & $\mathrm{N}$ & $\mathrm{N}$ & $\mathrm{N}$ & $\mathrm{N}$ & $\mathrm{N}$ & Y & $\mathrm{N}$ & Y & Y & 4 \\
\hline Ligibel JA, et al $(2012)^{* 30}$ & Y & N & Y & N & $\mathrm{N}$ & N & N & $\mathrm{N}$ & Y & Y & 4 \\
\hline Moffet $\mathrm{H}$, et al (2015) $)^{\star 26}$ & Y & Y & Y & $\mathrm{N}$ & $\mathrm{N}$ & Y & Y & Y & $Y$ & Y & 8 \\
\hline Morey MC, et al (2012) $)^{* 93}$ & Y & Y & Y & N & $\mathrm{N}$ & Y & Y & Y & Y & Y & 8 \\
\hline Morey MC, et al (2009) & Y & $\mathrm{N}$ & Y & $\mathrm{N}$ & $\mathrm{N}$ & Y & Y & $\mathrm{N}$ & Y & Y & 6 \\
\hline O'Brien J, et al (2017)*36 & Y & N & N & N & $\mathrm{N}$ & N & Y & Y & Y & Y & 5 \\
\hline Odole AC and OD Ojo (2013) $)^{* 41}$ & Y & N & Y & N & N & $\mathrm{N}$ & Y & $\mathrm{N}$ & Y & Y & 5 \\
\hline Pastora-Bernal JM, et al (2018) ${ }^{\star 37}$ & Y & $\mathrm{N}$ & Y & N & N & Y & Y & $\mathrm{N}$ & Y & Y & 6 \\
\hline Paul L, et al $(2014)^{\star 50}$ & $Y$ & $\mathrm{~N}$ & $Y$ & N & $\mathrm{N}$ & $\mathrm{N}$ & $Y$ & $\mathrm{~N}$ & Y & Y & 5 \\
\hline Paul L, et al $(2019)^{* 95}$ & Y & N & Y & N & $\mathrm{N}$ & Y & Y & Y & Y & Y & 7 \\
\hline Peng $X$, et al $(2018)^{* 63}$ & Y & Y & Y & $\mathrm{N}$ & $\mathrm{N}$ & Y & $\mathrm{N}$ & $\mathrm{N}$ & $\mathrm{Y}$ & Y & 6 \\
\hline Piga $\mathrm{M}$, et al (2014)*45 & Y & $\mathrm{N}$ & N & N & $\mathrm{N}$ & N & Y & N & Y & Y & 4 \\
\hline Piotrowicz E, et al $(2015)^{\star 70}$ & Y & $\mathrm{N}$ & Y & N & $\mathrm{N}$ & $\mathrm{N}$ & Y & $\mathrm{N}$ & Y & Y & 5 \\
\hline Piqueras $\mathrm{M}$, et al (2013) & Y & N & Y & N & N & Y & $\mathrm{N}$ & N & Y & Y & 5 \\
\hline Salvetti XM, et al (2008)*71 & Y & Y & Y & $\mathrm{N}$ & $\mathrm{N}$ & $\mathrm{N}$ & Y & $\mathrm{N}$ & Y & $\mathrm{Y}$ & 6 \\
\hline Sari D and L Khorshid (2009)* & Y & N & Y & N & N & N & $\mathrm{N}$ & N & Y & Y & 4 \\
\hline Stewart AV, et al $(2003)^{* 92}$ & Y & $\mathrm{N}$ & Y & N & $\mathrm{N}$ & Y & Y & $\mathrm{N}$ & Y & Y & 6 \\
\hline Tsai LL, et al (2017) ${ }^{* 60}$ & Y & Y & Y & N & N & Y & Y & Y & $\mathrm{Y}$ & $\mathrm{Y}$ & 8 \\
\hline
\end{tabular}



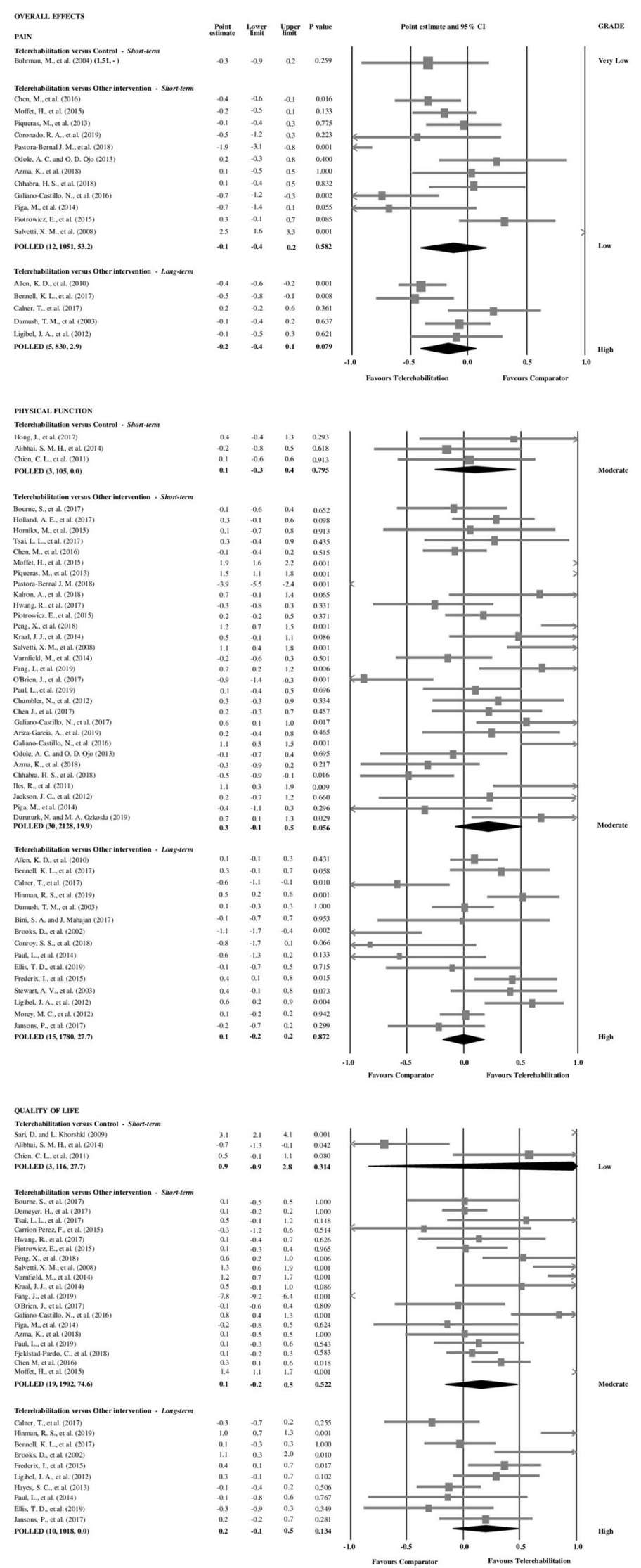

Figure 2 Overall effects of telerehabilitation on pain, physical function and quality of life. In parentheses: number of trials, total number of participants, $I^{2}$. Pain other intervention short-term: ( $Z=-0.5$, random-effects). Pain other intervention long-term: ( $Z=-1.8$, random-effects). Function control short-term: ( $Z=0.3$, random-effects). Physical function other intervention short-term: ( $Z=1.9$, random-effects). Physical function other intervention long-term: ( $Z=0.2$, random-effects). Quality of life control short-term: $(Z=1.0$, random-effects). Quality of life other intervention shortterm: ( $\mathrm{Z}=0.8$, random-effects). Quality of life other intervention long-term: $(\mathrm{Z}=1.5$, random-effects). Pain control short-term: individual trial. 

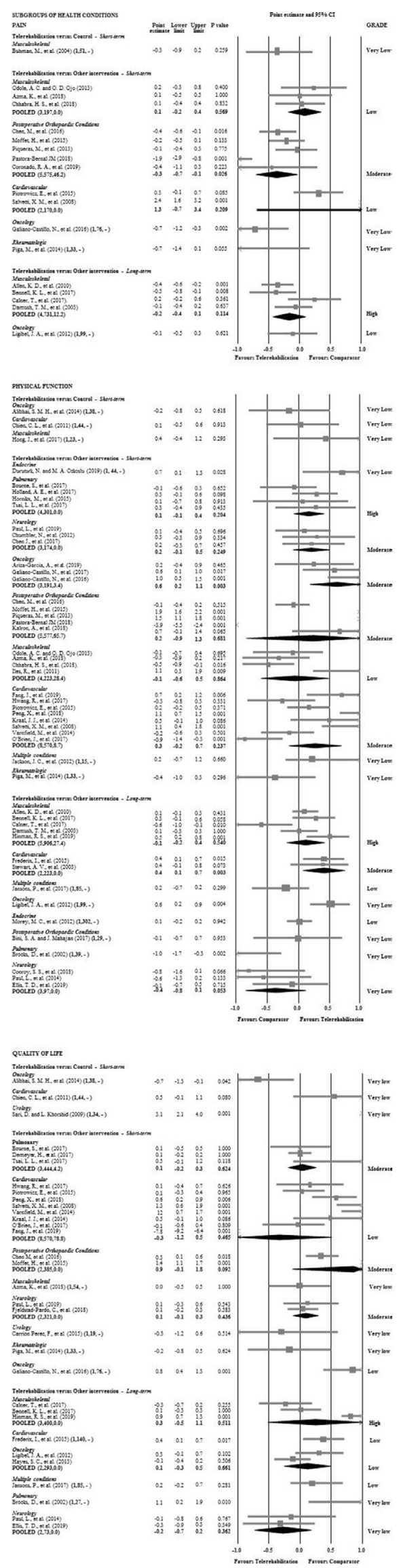

Figure 3 Subgroup analyses by clinical categories for pain, physical function and quality of life. In parentheses: number of trials, total number of participants, $I^{2}$. telerehabilitation was compared with control and with other intervention (figure 2).

\section{Physical function}

Overall effect analyses showed high-quality evidence that telerehabilitation was not different to other interventions on physical function at long-term (SMD of $0.195 \% \mathrm{CI}$ : -0.2 to $0.2 ; \mathrm{p}=0.872 ; 15$ trials $^{27} 2830464749-5165-67$ 91-94; $\left.\mathrm{n}=1780\right)$. At short-term, there was moderate evidence of no difference between telerehabilitation and control (SMD of 0.1 (95\% CI: -0.3 to $0.4 ; \mathrm{p}=0.795$; three trials $\left.{ }^{32} 52 ; \mathrm{n}=105\right)$ or other interventions (SMD of 0.3 (95\% CI: -0.1 to $0.5 ; \mathrm{p}=0.056 ; 30$ trials $^{26} 33-35$ 37-39 41-45 $\left.555658-6062-646769-71 \quad 87-90 \quad 9596 ; n=2128\right)$ (figure 2).

\section{Quality of life}

For quality of life, overall effect analyses showed high-quality evidence that telerehabilitation was not different to other interventions at long-term (SMD: $0.2 ; 95 \% \mathrm{CI}$ : -0.1 to 0.5 ; $\mathrm{p}=0.134 ; 10$ trials $\left.^{27293047505165-6794} ; \mathrm{n}=1018\right)$ and moderatequality evidence that telerehabilitation was not different to other interventions at short-term when compared with other intervention (SMD: $0.1 ; 95 \% \mathrm{CI}$ : -0.2 to $0.5 ; \mathrm{p}=0.522 ; 19$ trials $\left.^{2633} 3536404245555660-6469-718895 ; \mathrm{n}=1902\right)$. The strength of the recommendation was low when telerehabilitation was compared with control at short-term (figure 2).

Effects of telerehabilitation on pain, physical function and quality of life for different subgroups of health conditions

Subgroup analyses using meta-regression to investigate the impact of clinical categories on the overall effect estimates were possible only when telerehabilitation was compared with other intervention because of small number of pooled trials (ie, $<10$ trials): outcome of pain at short-term; physical function at shortterm and long-term; and quality of life at short-term. Qualitative analyses were conducted for the remained comparisons. Detailed subgroup analyses for all outcomes of interest are presented in figure 3 .

\section{Pain}

When compared with other interventions at short-term, results of meta-regression showed impact of clinical categories on overall estimates $(p<0.001)$. Qualitative subgroup analyses by clinical categories also suggested impact of subgroups on the overall estimates for pain. There was high-quality evidence of no difference between telerehabilitation and other intervention on pain at long-term for musculoskeletal conditions (SMD: -0.2 ; 95\% CI: -0.4 to $0.1 ; \mathrm{p}=0.114$; four trials ${ }^{272846} ; \mathrm{n}=731$ ) and moderate-quality evidence a small effect of telerehabilitation for postoperative orthopaedic conditions at short-term (SMD: $-0.3 ; 95 \% \mathrm{CI}:-0.7$ to $-0.1 ; \mathrm{p}=0.026$; five trials ${ }^{26} 33343797$; $\mathrm{n}=575)$. The strength of the recommendation was low and very low for all the other comparisons (figure 3).

\section{Physical function}

When compared with other interventions at short-term and long-term, results of meta-regression showed impact of clinical categories on overall estimates for physical function $(\mathrm{p}<0.001)$. High-quality evidence showed that telerehabilitation was not different to other interventions on physical function for pulmonary conditions at short-term and for musculoskeletal conditions at long-term. SMDs of 0.1 (95\% CI: -0.1 to $0.4 ; \mathrm{p}=0.204$; four trials $\left.{ }^{5556860} ; \mathrm{n}=301\right)$ and -0.1 (95\% CI: -0.2 to 0.4 ; 
$\mathrm{p}=0.540$; five trials ${ }^{27} 28464794 ; \mathrm{n}=906$ ) for pulmonary and musculoskeletal conditions, respectively. Besides, moderatequality evidence showed a medium effect of telerehabilitation for oncology conditions at short-term (SMD: 0.6; 95\% CI: 0.2 to 1.1; $\mathrm{p}=0.003$; three trials ${ }^{69}{ }^{90}{ }^{96} ; \mathrm{n}=191$ ), a small effect of telerehabilitation for cardiovascular conditions at long-term (SMD: $0.4 ; 95 \% \mathrm{CI}: 0.1$ to $0.7 ; \mathrm{p}=0.003$; two trials ${ }^{51}{ }^{92}$; $\mathrm{n}=223$ ) and not different to other interventions at short-term for neurological (SMD: $0.2 ; 95 \%$ CI: -0.1 to $0.5 ; \mathrm{p}=0.249$; three trials ${ }^{38} 3995 ; \mathrm{n}=174$ ), cardiovascular conditions (SMD: $0.3 ; 95 \% \mathrm{CI}:-0.2$ to $0.7 ; \mathrm{p}=0.237$; eight trials ${ }^{353662-64707188}$; $\mathrm{n}=570$ ) and postoperative orthopaedic conditions (SMD: 0.2; 95\% CI: -0.9 to $1.3 ; \mathrm{p}=0.681$; five trials ${ }^{2633} 343789 ; \mathrm{n}=577$ ). As shown in figure 3 , low to very-low quality evidence also suggested impact of different subgroups of health conditions on the estimates for physical function.

\section{Quality of life}

When compared with other interventions at short-term, metaregression showed impact of clinical categories on overall estimates for quality of life $(\mathrm{p}<0.001)$. High-quality evidence showed that telerehabilitation was not different to other interventions on quality of life for musculoskeletal conditions at long-term (SMD: $0.3 ; 95 \% \mathrm{CI}$ : -0.5 to $1.1 ; \mathrm{p}=0.511$; three trials $^{274794} ; n=400$ ). Besides, moderate-quality evidence showed that telerehabilitation was not different to other interventions on quality of life at short-term for pulmonary, neurology and postoperative orthopaedic conditions. SMDs of 0.1 (95\% CI: -0.2 to $0.3 ; \mathrm{p}=0.624$; three trials $\left.{ }^{555760} ; \mathrm{n}=444\right), 0.1(95 \% \mathrm{CI}$ : -0.1 to $0.3 ; \mathrm{p}=0.436$; two trials $\left.{ }^{40} 95 \mathrm{n}=321\right)$ and $0.9(95 \%$ CI: -0.1 to $1.8 ; \mathrm{p}=0.092$; two trials ${ }^{2633} ; \mathrm{n}=385$ ), respectively. Qualitative subgroup analyses suggested impact of subgroups on the remained comparisons for quality of life as well.

\section{Sensitivity analysis}

Meta-regression to investigate the impact of methodological issues was possible for few cases when telerehabilitation was compared with other interventions: pain at short-term; physical function at short- and long-term; and quality of life at shortterm. Meta-regression showed impact of poor methods quality on overall estimates for pain at short-term, physical function at short-term and long-term and quality of life at short-term and long-term $(\mathrm{p}<0.001)$. Detailed qualitative sensitivity analyses by removing trials of poor methodological quality $(<6$ on the 0 to 10 PEDro scale) suggesting potential impact of poor methodological quality of included trials are presented in online supplemental material 5.

\section{DISCUSSION}

To our knowledge, this is the first systematic review with metaanalysis that investigated the effects of telerehabilitation on pain, physical function and quality of life in people with physical disabilities, when compared with control and other interventions. High-quality or moderate-quality evidence showed that telerehabilitation was not different to other interventions on pain at long-term, physical function at short-term and long-term and quality of life at short-term and long-term. Therefore, we are confident that the true effect lies close to the estimate of the effect and that telerehabilitation may be an alternative to treat people with physical disabilities. We have very little confidence in the effect estimate when telerehabilitation was compared with control.
In some included trials, telerehabilitation groups received more follow-up than the comparison groups, with more elaborate interventions preceded by conventional rehabilitation or periodic meetings during the intervention period. Hailey et $a l^{98}$ pointed out in their review on telerehabilitation in routine care that, in most studies, telerehabilitation intervention was more elaborate than the comparator, with additional services and more frequent contacts between patients and professionals. Thus, the authors argue that the positive results found could be attributed to the use of more elaborate interventions. Moreover, some trials investigated interventions focussed not only on the exercise protocol, but incorporated other strategies such as a stimulus to increase physical activity, self-management, education and behavioural changes. ${ }^{25} 27283143$ 46-48 5973 Multicomponent interventions have been employed in different contexts to facilitate self-management of the disease and to involve the patient in their treatment. ${ }^{97} 99100$ This type of intervention has shown better results when compared with single component interventions in chronic patients. ${ }^{100}$ Pietrzak et al ${ }^{101}$ identified in their review that self-management programmes, education and exercises at a distance can be used successfully in patients with osteoarthritis, resulting in improvements in health status indicators, access to care and communication between patients and health professionals. To investigate whether different types and dosage of exercise by telerehabilitation would impact on estimates, we planned subgroup analyses; however, investigation was not possible because of the small number of included trials.

Overall, for the outcomes of physical function and quality of life, our results showed evidence of moderate and high quality for no difference between telerehabilitation and other interventions at short-term and long-term. Therefore, it is likely that telerehabilitation is equivalent to other forms of care. Possible mediating variables reinforced the beneficial effects that physical activity exerts on quality of life. Self-efficacy in older adults, for example, is a possible mediator of physical and psychological results associated with physical activity, by increasing the sense of control and satisfaction with the lives of these individuals. ${ }^{102}$ Specific studies of cardiac populations have shown similar results. Hwang et al ${ }^{103}$ reported in their systematic review on the effects of telerehabilitation in patients with cardiopulmonary diseases that, in general, the telerehabilitation group significantly improved the quality of life of patients with cardiomyopathy. Chan et al ${ }^{104}$ conducted a meta-analysis on exercise by telemonitoring and telerehabilitation compared with traditional cardiac and pulmonary rehabilitation. They concluded that, for patients with cardiac diseases, telerehabilitation provided similar benefits to usual care and without reports of adverse effects.

\section{Efficacy}

Finally, due to the low-quality evidence and the small number of trials comparing exercise protocols offered by telerehabilitation with control groups, it is still not possible to state the efficacy of telerehabilitation on pain, function and quality of life at short-term and long-term, for adults with physical disabilities. In general, evidence comparing telerehabilitation with control group without intervention was considered low or very low due to imprecision (grouping $<400$ participants), risk of bias (PEDro score $<6)$ and/or inconsistency $\left(\mathrm{I}^{2}>50 \%\right)$. Further high-quality trials comparing telerehabilitation with control to investigate efficacy on our population of interest are needed. It is also promising in postoperative orthopaedic, oncological, cardiovascular, pulmonary, neurological and musculoskeletal conditions. 
The risk of bias of the trials was relatively low, with PEDro greater than 6 points out of 10 in more than half of the trials included in this review. This type of study, in recent years, has followed detailed guidelines and strict criteria for its publication. It is noteworthy that none of the trials reached the maximum score, which can be explained by the difficulty of blinding the participants and therapists, due to the characteristics of the interventions implemented by telerehabilitation. Two other limitations found in $50 \%$ of the included trials were the absence of concealed allocation and intention-to-treat analysis. These strategies have been recommended to preserve the integrity of randomisation and prevent bias caused by loss of participants. ${ }^{105} 106$ Without these, the benefits of randomisation may be lost. ${ }^{106}$

\section{Limitations}

This study has some limitations. A potential limitation was the heterogeneity across trials (eg, different clinical conditions and different telerehabilitation delivery modes pooled and risk of bias). To solve this potential limitation, we conducted clinical conditions subgroup and sensitivity analyses to explore their impact on the estimates using meta-regression when possible. Consistent findings showed that clinical categories and risk of bias impact on estimates. Subgroup analyses for telerehabilitation delivery mode was not possible due to small number of included trials. Another potential limitation was that our included trials assessed the same outcome of interest but measured it in different validated ways. In this context, we used SMDs to conduct meta-analysis in the current review. Although weighted mean differences are better for interpretation, SMDs are also allowed and recommended by the Cochrane ${ }^{77}$ to pool data from different measurements. Other sources of heterogeneity were also potential limitations, such as type and dosage of telerehabilitation. Exploration of their potential impact on the estimates was limited by the number of included trials and by missing data. To decrease these other potential limitations, we used random-effect models for pooling and did not consider trials reporting data not normally distributed in the quantitative analyses. ${ }^{77}$ Future trials with greater sample sizes and appropriate reported data should further investigate impact of types and dosage of exercise by telerehabilitation in our population of interest.

\section{What is already known}

Telerehabilitation has the capacity to provide equitable access to individuals who do not have access to services, whether for geographical, physical or economic reasons.

- Telerehabilitation has the potential to improve quality of care, increase access to services and support health services.

\section{What are the new findings}

- Exercise by telerehabilitation can lead to clinical results similar to other interventions in improvement of pain, physical function and quality of life at short-points and long-points.

- Few studies compared exercise by telerehabilitation with control groups without any intervention, so it is still not possible to affirm the efficacy of telerehabilitation in reducing pain, function and quality of life in adults with physical disabilities.

\section{CONCLUSIONS}

This systematic review with meta-analysis was developed to support decision-making related to public policies and health programmes. Policies based on scientific evidence have ensured that decisions are based on the best available scientific evidence. This systematic review indicates that exercise by telerehabilitation has at least similar effects on pain, physical function and quality of life when compared with other interventions. However, efficacy is still limited by the scarcity of trials and low certainty of the current evidence.

Contributors JFD, VCO, PRTB, FCMSD, MCM, RNK, RAR and RFS contributed to planning, conduct and reporting of the work described in this article.

Funding This study was financed in part by the Coordenação de Aperfeiçoamento de Pessoal de Nível Superior - Brasil (CAPES) - Finance Code 001. We are also thankful to the State of Minas Gerais Funding Agency FAPEMIG.

Competing interests None declared.

Patient consent for publication Not required.

Provenance and peer review Not commissioned; externally peer reviewed.

\section{ORCID iD}

Renan Alves Resende http://orcid.org/0000-0002-1609-3278

\section{REFERENCES}

1 World Health Organization. World report on disability, 2011: 1-24

2 Mendes AdaCG, Sá DAde, Miranda GMD, et al. Assistência pública de saúde no contexto dA transição demográfica brasileira: exigências atuais $E$ futuras. Cad Saude Publica 2012;28:955-64

3 de Souza MAP, Dias JF, Ferreira FR, et al. Characteristics and functional demands of patients at a local rehabilitation network: analysis from first contact. Cienc e Saude Coletiva 2016.

4 Pinheiro RS, Viacava F, Travassos C, et al. Gênero, morbidade, acesso E utilização de serviços de saúde no Brasil. Ciênc. saúde coletiva 2002;7:687-707.

5 Darzi AJ, Officer A, Abualghaib 0, et al. Stakeholders' perceptions of rehabilitation services for individuals living with disability: a survey study. Health Qual Life Outcomes 2016;14:2.

6 Seelman KD, Hartman LM. Telerehabilitation: policy issues and research tools. Int J Telerehabil 2009;1:47-58.

7 Cooper RA, Fitzgerald SG, Boninger ML, et al. Telerehabilitation: expanding access to rehabilitation expertise. Proc IEEE Inst Electr Electron Eng 2001;89:1174-93.

8 Russell TG. Physical rehabilitation using telemedicine. J Telemed Telecare 2007; 13:217-20

9 Zampolini M, Todeschini E, Bernabeu Guitart M, Guitart MB, et al. Tele-rehabilitation: present and future. Ann Ist Super Sanita 2008;44:125-34.

10 Block VAJ, Pitsch E, Tahir P, et al. Remote physical activity monitoring in neurological disease: a systematic review. PLoS One 2016;11:e0154335.

11 Frederix I, Vanhees L, Dendale P, et al. A review of telerehabilitation for cardiac patients. J Telemed Telecare 2015;21:45-53.

12 Cottrell MA, Galea OA, O'Leary SP, et al. Real-time telerehabilitation for the treatment of musculoskeletal conditions is effective and comparable to standard practice: a systematic review and meta-analysis. Clin Rehabil 2017;31:625-38.

13 Shukla H, Nair SR, Thakker D. Role of telerehabilitation in patients following total knee arthroplasty: evidence from a systematic literature review and meta-analysis. J Telemed Telecare 2017;23:339-46.

14 Roux L, Pratt M, Tengs TO, et al. Cost effectiveness of community-based physical activity interventions. Am J Prev Med 2008;35:578-88.

15 Müller-Riemenschneider F, Reinhold T, Willich SN. Cost-effectiveness of interventions promoting physical activity. Br J Sports Med 2009:43:70-6.

16 Heath GW, Parra DC, Sarmiento OL, et al. Evidence-based intervention in physical activity: lessons from around the world. Lancet 2012;380:272-81.

17 Agostini M, Moja L, Banzi R, et al. Telerehabilitation and recovery of motor function: a systematic review and meta-analysis. J Telemed Telecare 2015:21:202-13.

18 Kairy D, Lehoux P, Vincent $C$, et al. A systematic review of clinical outcomes, clinical process, healthcare utilization and costs associated with telerehabilitation. Disabil Rehabil 2009;31:427-47

19 Moher D, Liberati A, Tetzlaff J, et al. Systematic reviews and meta-analyses: the PRISMA statement. Annu Intern Med 2009;151:264-9.

20 Cochrane Library. Cochrane CH for SR of I, 2019 entions. Available: http://handbook5-1.cochrane.org

21 World Health Organization. International classification of functioning, disability and health (ICF), 2001. Available: https://apps.who.int/iris/bitstream/handle/10665/ 42407/9241545429.pdf 
22 Price DD, McGrath PA, Rafii A, et al. The validation of visual analogue scales as ratio scale measures for chronic and experimental pain. Pain 1983;17:45-56.

23 Butland RJ, Pang J, Gross ER, et al. Two-, six-, and 12-minute walking tests in respiratory disease. Br Med J 1982:284:1607-8.

24 Brazier JE, Harper R, Jones NM, et al. Validating the SF-36 health survey questionnaire: new outcome measure for primary care. BMJ 1992;305:160-4.

25 Buhrman M, Fältenhag S, Ström L, et al. Controlled trial of internet-based treatment with telephone support for chronic back pain. Pain 2004;111:368-77.

26 Moffet H, Tousignant M, Nadeau S, et al. In-home telerehabilitation compared with face-to-face rehabilitation after total knee arthroplasty: a Noninferiority randomized controlled trial. J Bone Joint Surg Am 2015;97:1129-41.

27 Bennell K, Campbell P, Egerton T, et al. Telephone coaching to enhance a physiotherapist-prescribed home-based physical activity program for knee osteoarthritis: a randomised clinical trial. Osteoarthritis Cartilage 2016;24:S44-5.

28 Damush TM, Weinberger M, Perkins SM, et al. Randomized trial of a selfmanagement program for primary care patients with acute low back pain: shortterm effects. Arthritis Rheum 2003;49:179-86.

29 Hayes SC, Rye S, Disipio T, et al. Exercise for health: a randomized, controlled trial evaluating the impact of a pragmatic, translational exercise intervention on the quality of life, function and treatment-related side effects following breast cancer. Breast Cancer Res Treat 2013;137:175-86.

30 Ligibel JA, Meyerhardt J, Pierce JP, et al. Impact of a telephone-based physical activity intervention upon exercise behaviors and fitness in cancer survivors enrolled in a cooperative group setting. Breast Cancer Res Treat 2012;132:205-13.

31 Goode AP, Taylor SS, Hastings SN, et al. Effects of a home-based TelephoneSupported physical activity program for older adult veterans with chronic low back pain. Phys Ther 2018;98:369-80.

32 Hong J, Kim J, Kim SW, et al. Effects of home-based tele-exercise on sarcopenia among community-dwelling elderly adults: body composition and functional fitness. Exp Gerontol 2017;87:33-9.

33 Chen M, Li P, Lin F. Influence of structured telephone follow-up on patient compliance with rehabilitation after total knee arthroplasty. Patient Prefer Adherence 2016:10:257-64.

34 Piqueras $\mathrm{M}$, Marco $\mathrm{E}$, Coll $\mathrm{M}$, et al. Effectiveness of an interactive virtual telerehabilitation system in patients after total knee arthoplasty: a randomized controlled trial. J Rehabil Med 2013:45:392-6.

35 Kraal JJ, Peek N, Van den Akker-Van Marle ME, et al. Effects of home-based training with telemonitoring guidance in low to moderate risk patients entering cardiac rehabilitation: short-term results of the FIT@Home study. Eur J Prev Cardiol 2014:21:26-31.

36 O'Brien J, Finlayson K, Kerr G, et al. Evaluating the effectiveness of a selfmanagement exercise intervention on wound healing, functional ability and healthrelated quality of life outcomes in adults with venous leg ulcers: a randomised controlled trial. Int Wound J 2017;14:130-7.

37 Pastora-Bernal JM, Martín-Valero R, Barón-López FJ, Baro FJ, et al. Telerehabilitation after arthroscopic subacromial decompression is effective and not inferior to standard practice: preliminary results. J Telemed Telecare 2018;24:428-33.

38 Chumbler NR, Quigley P, Li X, et al. Effects of telerehabilitation on physical function and disability for stroke patients: a randomized, controlled trial. Stroke 2012;43:2168-74.

39 Chen J, Jin W, Dong WS, et al. Effects of home-based telesupervising rehabilitation on physical function for stroke survivors with hemiplegia: a randomized controlled trial. Am J Phys Med Rehabil 2017;96:152-60

40 Fjeldstad-Pardo C, Thiessen A, Pardo G. Telerehabilitation in multiple sclerosis: results of a randomized feasibility and efficacy pilot study. Int J Telerehabil 2018;10:55-64.

41 Odole AC, Ojo OD. A Telephone-based physiotherapy intervention for patients with osteoarthritis of the knee. Int J Telerehabil 2013;5:11-20.

42 Azma K, RezaSoltani Z, Rezaeimoghaddam F, et al. Efficacy of tele-rehabilitation compared with office-based physical therapy in patients with knee osteoarthritis: a randomized clinical trial. J Telemed Telecare 2018;24:560-5.

43 Iles R, Taylor NF, Davidson M, et al. Telephone coaching can increase activity levels for people with non-chronic low back pain: a randomised trial. J Physiother 2011;57:231-8.

44 Jackson JC, Ely EW, Morey MC, et al. Cognitive and physical rehabilitation of intensive care unit survivors. Crit Care Med 2012:40:1088-97.

45 Piga M, Tradori I, Pani D, et al. Telemedicine applied to kinesiotherapy for hand dysfunction in patients with systemic sclerosis and rheumatoid arthritis: recovery of movement and telemonitoring technology. J Rheumatol 2014;41:1324-33.

46 Allen KD, Oddone EZ, Coffman CJ, et al. Telephone-based self-management of osteoarthritis: a randomized trial. Ann Intern Med 2010;153:570-9.

47 Calner T, Nordin C, Eriksson MK, et al. Effects of a self-guided, web-based activity programme for patients with persistent musculoskeletal pain in primary healthcare: a randomized controlled trial. Eur J Pain 2017;21:1110-20.

48 Cuperus N, Hoogeboom TJ, Kersten CC, et al. Randomized trial of the effectiveness of a non-pharmacological multidisciplinary face-to-face treatment program on daily function compared to a telephone-based treatment program in patients with generalized osteoarthritis. Osteoarthr Cartil 2015;23:1267-75.
49 Bini SA, Mahajan J. Clinical outcomes of remote asynchronous telerehabilitation are equivalent to traditional therapy following total knee arthroplasty: a randomized control study. J Telemed Telecare 2017;23:239-47

50 Paul L, Coulter EH, Miller L, et al. Web-based physiotherapy for people moderately affected with multiple sclerosis; quantitative and qualitative data from a randomized, controlled pilot study. Clin Rehabil 2014;28:924-35.

51 Frederix I, Hansen D, Coninx K, et al. Medium-term effectiveness of a comprehensive Internet-based and patient-specific Telerehabilitation program with text messaging support for cardiac patients: randomized controlled trial. J Med Internet Res 2015; 17:e185-15.

52 Alibhai SMH, O'Neill S, Fisher-Schlombs K, et al. A pilot phase II RCT of a home-based exercise intervention for survivors of AML. Support Care Cancer 2014;22:881-9.

53 Chien C-L, Lee C-M, Wu Y-W, et al. Home-based exercise improves the quality of life and physical function but not the psychological status of people with chronic heart failure: a randomised trial. J Physiother 2011;57:157-63.

54 Sar D, Khorshid $\mathrm{L}$. The effects of pelvic floor muscle training on stress and mixed urinary incontinence and quality of life. J Wound Ostomy Continence Nurs 2009:36:429-35.

55 Bourne S, DeVos R, North M, et al. Online versus face-to-face pulmonary rehabilitation for patients with chronic obstructive pulmonary disease: randomised controlled trial. BMJ Open 2017;7:e014580

56 Demeyer $\mathrm{H}$, Louvaris $\mathrm{Z}$, Frei $\mathrm{A}$, et al. Physical activity is increased by a 12-week semiautomated telecoaching programme in patients with COPD: a multicentre randomised controlled trial. Thorax 2017:72:415-23.

57 Holland AE, Mahal A, Hill CJ, et al. Home-based rehabilitation for COPD using minimal resources: a randomised, controlled equivalence trial. Thorax 2017;72:57-65

58 Hornikx M, Demeyer H, Camillo CA, et al. The effects of a physical activity counseling program after an exacerbation in patients with chronic obstructive pulmonary disease: a randomized controlled pilot study. BMC Pulm Med 2015; 15:136.

59 Chhabra HS, Sharma S, Verma S. Smartphone APP in self-management of chronic low back pain: a randomized controlled trial. Eur Spine J 2018;27:2862-74.

60 Tsai LLY, McNamara RJ, Moddel C, et al. Home-based telerehabilitation via real-time videoconferencing improves endurance exercise capacity in patients with COPD: the randomized controlled TeleR study. Respirology 2017;22:699-707.

61 Carrion Perez F, Moreno MR, Carnerero Cordoba L, et al. Tratamiento de la incontinencia urinaria de esfuerzo mediante telerrehabilitacion. Estudio piloto 2015;144:445-8.

62 Hwang R, Bruning J, Morris NR, et al. Home-based telerehabilitation is not inferior to a centre-based program in patients with chronic heart failure: a randomised trial. $J$ Physiother 2017;63:101-7.

63 Peng $X$, Su Y, Hu Z, et al. Home-based telehealth exercise training program in Chinese patients with heart failure: a randomized controlled trial. Medicine 2018:97:e12069.

64 Varnfield M, Karunanithi M, Lee C-K, et al. Smartphone-based home care model improved use of cardiac rehabilitation in postmyocardial infarction patients: results from a randomised controlled trial. Heart 2014;100:1770-9.

65 Brooks D, Krip B, Mangovski-Alzamora S, et al. The effect of postrehabilitation programmes among individuals with chronic obstructive pulmonary disease. Eur Respir J 2002;20:20-9.

66 Ellis TD, Cavanaugh JT, DeAngelis T, et al. Comparative effectiveness of mHealthSupported exercise compared with exercise alone for people with Parkinson disease: randomized controlled pilot study. Phys Ther 2019;99:203-16.

67 Jansons P, Robins L, O'Brien L, et al. Gym-based exercise and home-based exercise with telephone support have similar outcomes when used as maintenance programs in adults with chronic health conditions: a randomised trial. J Physiother 2017:63:154-60.

68 Bernocchi P, Vitacca M, La Rovere MT, et al. Home-based telerehabilitation in older patients with chronic obstructive pulmonary disease and heart failure: a randomised controlled trial. Age Ageing 2018;47:82-8.

69 Galiano-Castillo N, Cantarero-Villanueva I, Fernández-Lao C, et al. Telehealth system: a randomized controlled trial evaluating the impact of an Internet-based exercise intervention on quality of life, pain, muscle strength, and fatigue in breast cancer survivors. Cancer 2016;122:3166-74.

70 Piotrowicz E, Stepnowska M, Leszczyńska-Iwanicka K, et al. Quality of life in heart failure patients undergoing home-based telerehabilitation versus outpatient rehabilitation--a randomized controlled study. Eur J Cardiovasc Nurs 2015;14:256-63

71 Salvetti XM, Oliveira JA, Servantes DM, et al. How much do the benefits cost? effects of a home-based training programme on cardiovascular fitness, quality of life, programme cost and adherence for patients with coronary disease. Clin Rehabil 2008:22:987-96

72 Morey MC, Snyder DC, Sloane R, et al. Effects of home-based diet and exercise on functional outcomes among older, overweight long-term cancer survivors: RENEW: a randomized controlled trial. JAMA 2009;301:1883-91. 
73 Allen KD, Yancy WS, Bosworth HB, et al. A combined patient and provider intervention for management of osteoarthritis in veterans: a randomized clinical trial. Ann Intern Med 2016;164:73.

74 Macedo LG, Elkins MR, Maher CG, et al. There was evidence of convergent and construct validity of physiotherapy evidence database quality scale for physiotherapy trials. J Clin Epidemiol 2010;63:920-5.

75 Maher CG, Sherrington C, Herbert RD, et al. Reliability of the PEDro scale for rating quality of randomized controlled trials. Phys Ther 2003:83:713-21.

76 de Morton NA. The PEDro scale is a valid measure of the methodological quality of clinical trials: a demographic study. Aust J Physiother 2009;55:129-33.

77 Higgins JP GS. Cochrane handbook for systematic reviews of interventions, version 5.1.0, 2011. http://handbook.cochrane.org/

78 Altman DG, Bland JM, Airman DG, Martin Bland J. Detecting skewness from summary information. BMJ 1996;313:1200.

79 Sampaio RFet al. Aplicação dA Classificação Internacional de Funcionalidade, Incapacidade E Saúde (CIF) Na Prática Clínica do Fisioterapeuta. Rev Bras Fisioter2005.

80 Organização Mundial da Saúde. CID-10 classificação estatística internacional de doenças E problemas relacionados saúde, 1997.

81 Ioannidis JPA, Trikalinos TA. The appropriateness of asymmetry tests for publication bias in meta-analyses: a large survey. CMAJ 2007;176:1091-6.

82 Bosco FA, Aguinis H, Singh K, et al. Correlational effect size benchmarks. J App/ Psychol 2015;100:431-49.

83 Guyatt G, Oxman AD, Akl EA, et al. Grade guidelines: 1. introduction-GRADE evidence profiles and summary of findings tables. J Clin Epidemiol 2011;64:383-94.

84 Guyatt $\mathrm{GH}$, Oxman AD, Kunz R, et al. GRADE guidelines 6 . rating the quality of evidence--imprecision. J Clin Epidemiol 2011;64:1283-93.

85 Guyatt GH, Oxman AD, Vist G, et al. GRADE guidelines: 4. rating the quality of evidence - study limitations (risk of bias). J Clin Epidemiol 2011.

86 Guyatt GH, Oxman AD, Kunz R, et al. GRADE guidelines: 7. rating the quality of evidence--inconsistency. J Clin Epidemiol 2011;64:1294-302.

87 Duruturk N, Özköslü MA. Effect of tele-rehabilitation on glucose control, exercise capacity, physical fitness, muscle strength and psychosocial status in patients with type 2 diabetes: a double blind randomized controlled trial. Prim Care Diabetes 2019;13:542-8.

88 Fang J, Huang B, Xu D, et al. Innovative application of a home-based and remote sensing cardiac rehabilitation protocol in Chinese patients after percutaneous coronary intervention. Telemed J E Health 2019;25:288-93.

89 Kalron A, Tawil H, Peleg-Shani S, et al. Effect of telerehabilitation on mobility in people after hip surgery: a pilot feasibility study. Int J Rehabil Res 2018;41:244-50.

90 Galiano-Castillo N, Arroyo-Morales M, Lozano-Lozano M, et al. Effect of an Internetbased telehealth system on functional capacity and cognition in breast cancer survivors: a secondary analysis of a randomized controlled trial. Support Care Cancer 2017:25:3551-9.
91 Conroy SS, Zhan M, Culpepper WJ, et al. Self-directed exercise in multiple sclerosis: evaluation of a home automated tele-management system. J Telemed Telecare 2018;24:410-9.

92 Stewart AV, EALES CJ, DAVIS KA. Effect of a telephonic intervention on the adherence of patients with hypertension.. Sa Journal of Physiotherapy 2003;59.

93 Morey MC, Pieper CF, Edelman DE, et al. Enhanced fitness: a randomized controlled trial of the effects of home-based physical activity counseling on glycemic control in older adults with prediabetes mellitus. J Am Geriatr Soc 2012;60:1655-62.

94 Hinman RS, Campbell PK, Lawford BJ, et al. Does telephone-delivered exercise advice and support by physiotherapists improve pain and/or function in people with knee osteoarthritis? Telecare randomised controlled trial. Br J Sports Med 2020;54:790-7.

95 Paul L, Renfrew L, Freeman J, et al. Web-based physiotherapy for people affected by multiple sclerosis: a single blind, randomized controlled feasibility study. Clin Rehabil 2019:33:473-84.

96 Ariza-Garcia A, Lozano-Lozano M, Galiano-Castillo N, et al. A web-based exercise system (e-cuidatechemo) to counter the side effects of chemotherapy in patients with breast cancer: randomized controlled trial. J Med Internet Res 2019;21:e14418.

97 Coronado RA, Devin CJ, Pennings JS, et al. Early Self-directed home exercise program after anterior cervical discectomy and fusion: a pilot study. Spine 2020;45:217-25.

98 Hailey D, Roine R, Ohinmaa A, et al. Evidence of benefit from telerehabilitation in routine care: a systematic review. J Telemed Telecare 2011;17:281-7.

99 Cheng S-T, Chan KL, Lam RWL, et al. A multicomponent intervention for the management of chronic pain in older adults: study protocol for a randomized controlled trial. Trials 2017; 18:528.

100 Carnes D, Homer K, Miles C, et al. T524 effective delivery styles and content for chronic pain self-management interventions: a systematic review. Eur J Pain Supp/ 2011:5:80.

101 Pietrzak E, Cotea C, Pullman S, et al. Self-Management and rehabilitation in osteoarthritis: is there a place for Internet-based interventions? Telemed J E Health 2013;19:800-5

102 Rejeski WJ, Mihalko SL. Physical activity and quality of life in older adults. J Gerontol A Biol Sci Med Sci 2001;56 Spec No 2:23-35.

103 Hwang R, Bruning J, Morris N, et al. A systematic review of the effects of telerehabilitation in patients with cardiopulmonary diseases. J Cardiopulm Rehabil Prev 2015;35:380-9.

104 Chan C, Yamabayashi C, Syed N, et al. Exercise telemonitoring and telerehabilitation compared with traditional cardiac and pulmonary rehabilitation: a systematic review and meta-analysis. Physiother Can 2016;68:242-51.

105 Moher D, Hopewell S, Schulz KF, et al. Consort 2010 explanation and elaboration: updated guidelines for reporting parallel group randomised trials. Int I Surg 2012;10:28-55.

106 Berwanger O, Avezum A, Guimarães HP. The ten commandments of the randomized clinical trial - principles for critical appraisal of the medical literature, 2006: 65-70. 